Received 05/23/2017

Review began 05/28/2017

Review ended 05/30/2017

Published 06/05/2017

C Copyright 2017

Cox et al. This is an open access article distributed under the terms of the Creative Commons Attribution License CC-BY 3.0., which permits unrestricted use, distribution, and reproduction in any medium, provided the original author and source are credited.

\title{
Pineal Gland Agenesis: Review and Case Illustration
}

\author{
Marcus A. Cox ${ }^{1}$, Michele Davis ${ }^{2}$, Vlad Voin ${ }^{3}$, Mohammadali M. Shoja ${ }^{4}$, Rod J. Oskouian ${ }^{5}$, \\ Marios Loukas ${ }^{6}$, R. Shane Tubbs ${ }^{7}$ \\ 1. Internal Medicine, Saint Michael's Medical Center 2. Department of Anatomical Sciences, St. George's \\ University School of Medicine, Grenada, West Indies 3. Research Fellow, Seattle Science Foundation 4. \\ Department of Surgery, University of Texas Medical Branch at Galveston 5. Swedish Neuroscience \\ Institute 6. Department of Anatomical Sciences, St. George's University School of Medicine, Grenada, \\ West Indies 7. Neurosurgery, Seattle Science Foundation
}

$\square$ Corresponding author: Michele Davis, mdavis4@sgu.edu

Disclosures can be found in Additional Information at the end of the article

\section{Abstract}

Agenesis of the pineal gland has rarely been reported in the medical literature. Herein, we report a cadaveric specimen found to have agenesis of the pineal gland. The remaining gross examination of the brain was normal. A review of the literature was performed on this unusual finding.

Categories: Endocrinology/Diabetes/Metabolism, Neurology, Neurosurgery

Keywords: pineal gland, embryology, brain, diencephalon, aplasia, variation

\section{Introduction}

The pineal gland is a neuroendocrine organ which develops from the diencephalon. Although the primary function is the secretion of melatonin, the role in human growth and development has not been clearly defined. Although exceptionally rare, the absence of the pineal gland has been recorded as an incidental finding on magnetic resonance imaging (MRI). It has been linked to the PAX6 gene mutation, which is a transcription factor [1-2]. In this article, we will review rare cases of pineal agenesis and their associated syndromes, with special attention to ocular and brain abnormalities in patients with PAX6 mutations. A cadaveric specimen with pineal gland agenesis will be presented as an illustration.

\section{Case Presentation}

During routine dissection of a Caucasian male cadaver (79 years old at death), the absence of the pineal gland was noted (Figure 1). The patient had a history of hypertension, osteoarthritis, and glaucoma. He expired due to a myocardial infarction. No other medical history was reported including any syndromes or previous intracranial surgery. The patient had a left inguinal region incision and had undergone a left knee replacement. 


\section{Cureus}

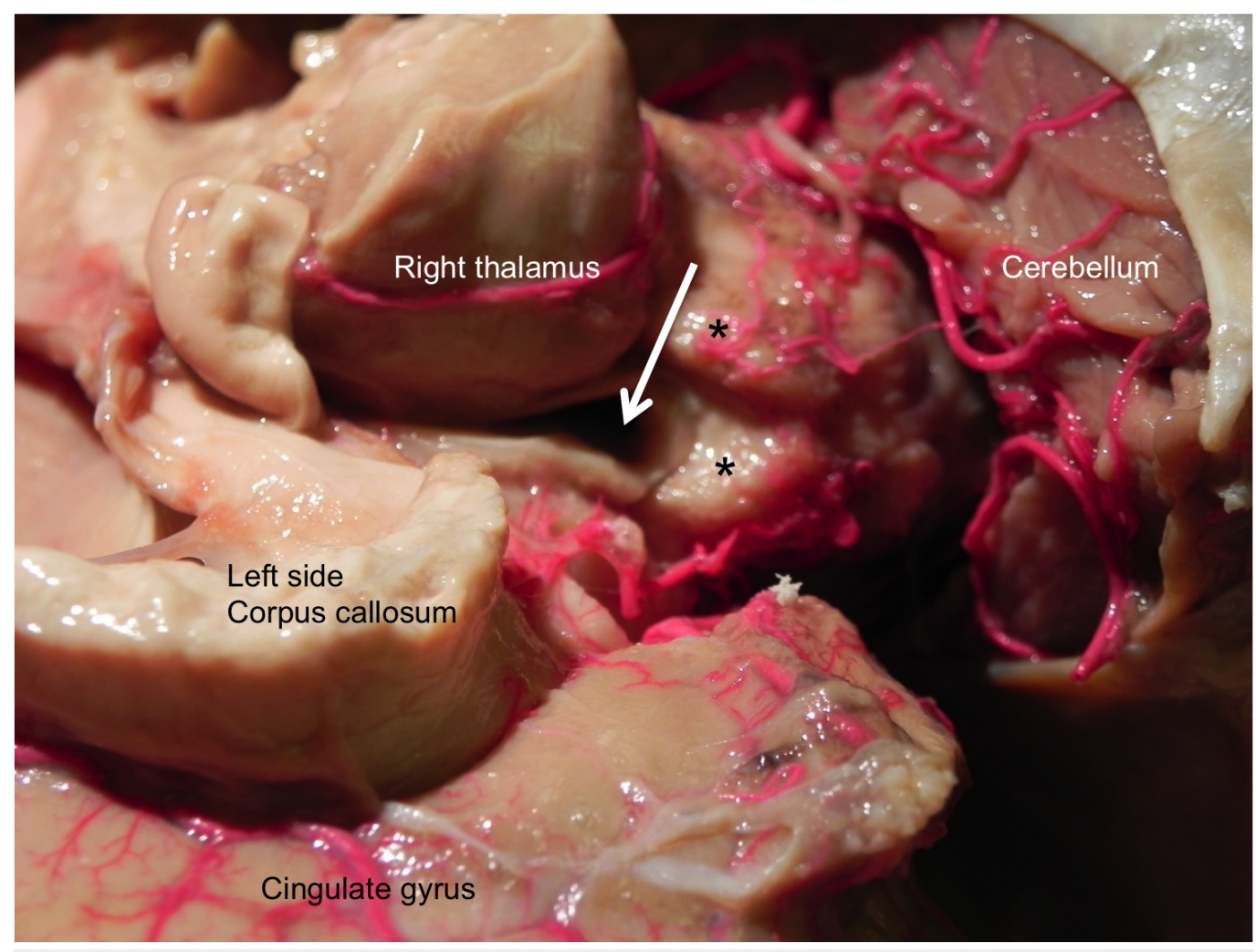

\section{FIGURE 1: Absent Pineal Gland}

Left posterolateral view of the brain of the case illustration herein. Notice the absence of the pineal gland.

During the dissection of the cranium and its contents, no other intracranial anatomical variations were noted. Specifically, the other diencephalic derived brain structures (e.g., thalamus, optic nerves, hypothalamus, posterior pituitary gland) were all within normal limits. No pathology of the brain was noted such as hydrocephalus or ectopic tissues.

\section{Discussion}

The pineal gland develops as an evagination of the neuroepithelium from the dorsal diencephalon, above the future third ventricle, and has been attributed as the main producer of melatonin. The shape of the pineal gland in mammals is variable but usually presents as a lobular structure. From early human life the pineal gland begins to calcify and calcification increases with age, which may correlate with the age-related decline in melatonin production. Decreases in the night time levels of this hormone may be responsible for fragmented sleepwake patterns [3].

Pineal gland agenesis has been observed in murine and humans with mutations in the paired box gene 6 (PAX6), where the pineal gland along with other brain and eye abnormalities has been observed [1, 4-5]. PAX6 is expressed in the telencephalon, diencephalon, caudal part of the rhombencephalon, myelencephalon, the spinal cord and pancreas, explaining the various phenotypes observed in individuals with mutations in this gene [1, 4-5]. PAX6 has been shown to play an important role as a regulatory gene in cortical developmental processes including cellular proliferation, neuronal migration, and axonal guidance [1, 6-7]. The mechanism by which PAX6 expression influences structural formation is unknown, but one explanation is that PAX6 regulates expression of a gene directing synthesis of R-cadherin and other cell surface 
recognition molecules [8]. Mapping results of the PAX6 gene by Thakurela, et al., demonstrated a dual function of PAX6 upon neuronal commitment where it mediates the activation of neuronal genes while concurrently suppressing the mesodermal and endodermal genes to ensure the unidirectionality toward neuronal differentiation [9]. They also concluded that PAX6 induces critical signaling pathways that further work together in guiding critical neurogenic events such as neurogenesis, neuronal differentiation, and lens development [9].

In mice, PAX6 is critical for survival. PAX6 null mice die immediately after birth [5-6]. Heterozygote mice have a subtle form of cortical and eye abnormalities. There have only been a few cases reported in humans with mutations in both PAX6 alleles. In these cases, patients had severe brain and eye abnormalities reported $[5,7]$. Human heterozygotes have a variety of anomalies [1-2, 6-7]. Studies have been performed using imaging techniques in patients with PAX6 mutation to identify the extent of these abnormalities.

High-resolution MRIs performed on 24 individuals with ocular abnormalities and positive PAX6 mutations showed 13 individuals had absent pineal glands (54\%), four had structurally normal pineal glands (17\%) and the remaining seven had pineal glands which were difficult to visualize and were deemed "hypoplastic" (29\%) [1]. Although this study shows a link between PAX6 mutations, aniridia, and pineal agenesis, it is limited by the small sample size and the possibility that pineal tissue may be demonstrable histopathologically while not being visualized on MRIs. Abouzeid, et al. showed that out of 10 patients from three different families in Egypt who had PAX6 mutations, all 10 had bilateral aniridia while three had absent pineal glands (30\%) [4]. This was much less than the $54 \%$ in the study of Mitchell, et al. who had both pineal agenesis and ocular abnormalities [1]. All patients in the study published by Abouzeid, et al. had a full ophthalmic and neurological examinations performed at the time and showed no major neurodevelopmental delays [4]. Although the sample size remains small, the current evidence points to a distinct link between ocular abnormalities and pineal agenesis with PAX6 mutations.

Yogarajah, et al. investigated MRI parameters in 19 adults with known PAX6 mutations to understand the importance that the PAX6 gene plays in the maintenance of brain integrity. They found in people with heterozygous mutation in PAX6, there was exaggerated cortical thinning with age, a reduction in cortical thickness that correlated with a decline in working memory, and abnormalities of cortical patterning. They concluded that these findings could play an important role in understanding neurodegenerative disorders and modulation of PAX6 may offer new therapeutic strategies in fighting these diseases [5].

Ellison-Wright, et al. demonstrated significant reductions of white matter in the anterior and posterior corpus callosum, local excesses of grey matter in regions including the hippocampus, ventral striatum, insula, and cerebellum in humans with heterozygous PAX6 mutation [8]. Free, et al. established variations in cortical structures in patients with a PAX6 abnormality using quantitative MRI analysis [7]. Sisodiya, et al. observed that patients with the PAX6 mutation had cerebral malformation and olfactory dysfunctions [10]. These findings show there is a link between people with PAX6 mutations and abnormal brain development.

Being a transcription factor, PAX6 interacts with several brain developmental genes and other transcription factors. With the development of high-resolution MRIs, the extent of abnormalities in these patients is being examined more closely.

\section{Conclusions}

The extent to which pineal agenesis affects human growth and development has not been explored in the articles reviewed. Extensive neurological, endocrine, and sleep studies on these 
patients might help provide further insight into the function of this gland in normal human growth and development. Also, the PAX6 gene plays an important role in brain development and formation. While the correlation between PAX6 gene mutation and pineal agenesis is not definite, there seems to be a link between patients with pineal agenesis and mutations in the PAX6 gene. Further study of this gene could lead to novel treatments and our understanding of neurodegenerative diseases.

\section{Additional Information \\ Disclosures}

Human subjects: All authors have confirmed that this study did not involve human participants or tissue. Conflicts of interest: In compliance with the ICMJE uniform disclosure form, all authors declare the following: Payment/services info: All authors have declared that no financial support was received from any organization for the submitted work. Financial relationships: All authors have declared that they have no financial relationships at present or within the previous three years with any organizations that might have an interest in the submitted work. Other relationships: All authors have declared that there are no other relationships or activities that could appear to have influenced the submitted work.

\section{References}

1. Mitchell TN, Free SL, WIlliamson KA, et al.: Polymicrogyria and absence of pineal gland due to PAX6 mutation. Ann Neurol. 2003, 53:658-663. 10.1002/ana.10576

2. Al-Owain M, Al-Zahrani J, Al-Bakhett A, et al.: A novel syndrome of abnormal striatum and congenital cataract: evidence for linkage to chromosomes 11. Clin Genet. 2013, 84:258-264. 10.1111/cge.12066

3. Sapède D, Cau E: The pineal gland from development to function . Curr Top Dev Biol. 2013, 106:171-215. 10.1016/B978-0-12-416021-7.00005-5

4. Abouzeid H, Youssef MA, ElShakankiri N, et al.: PAX6 aniridia and interhemispheric brain anomalies. Mol Vis. 2009, 15:2074-2083.

5. Yogarajah M, Matarin M, Vollmar C, et al.: PAX6, brain structure and function in human adults: advanced MRI in aniridia. Ann Clin Transl Neurol. 2016, 3:314-330. 10.1002/acn3.297

6. Thompson PJ, Mitchell TN, Free SL, et al.: Cognitive functioning in humans with mutations of the PAX6 gene. Neurology. 2004, 62:1216-1218. 10.1212/01.WNL.0000118298.81140.62

7. Free SL, Mitchell TN, Williamson KA, et al.: Quantitative MR image analysis in subjects with defects in the PAX6 gene. Neuroimage. 2003, 20:2281-2290.

10.1016/j.neuroimage.2003.07.001

8. Ellison-Wright Z, Heyman I, Frampton I, et al.: Heterozygous PAX6 mutation, adult brain structure and fronto-striato-thalamic function in a human family. Eur J Neurosci. 2004, 19:1505-1512. 10.1111/j.1460-9568.2004.03236.x

9. Thakurela S, Tiwari N, Schick S, et al.: Mapping gene regulatory circuitry of Pax6 during neurogenesis. Cell Discov. 2016, 2:15045. 10.1038/celldisc.2015.45

10. Sisodiya SM, Free SL, Williamson KA, et al.: PAX6 haploinsufficiency causes cerebral malformation and olfactory dysfunction in humans. Nat Genet. 2001, 28:214-216.

$10.1038 / 90042$ 